\title{
Sphingolipids and cell signaling: Relationship between health and disease in the central nervous system
}

Andrés Felipe Leal ${ }^{1}$, Diego A. Suarez ${ }^{1,2}$, Olga Yaneth Echeverri-Peña ${ }^{1}$, Sonia Luz Albarracín ${ }^{3}$, Carlos Javier Alméciga-Díaz ${ }^{1 *}$, Angela Johana Espejo-Mojica ${ }^{*}$

1 Institute for the Study of Inborn Errors of Metabolism, Faculty of Science, Pontificia Universidad Javeriana, Bogotá D.C., 110231, Colombia; lealb.af@javeriana.edu.co (A.F.L.), suarezd.i@javeriana.edu.co (D.A.S.), oyecheve@javeriana.edu.co (O.Y.E.P.)

2 Faculty of Medicine, Universidad Nacional de Colombia, Bogotá D.C., Colombia; dasuarezg@unal.edu.co (D.A.S.)

3 Nutrition and Biochemistry Department, Faculty of Science, Pontificia Universidad Javeriana, Bogotá D.C., Colombia; albarra@javeriana.edu.co (S.L.A.)

* Correspondence: $\quad$ cjalmeciga@javeriana.edu.co; $\quad$ Tel.: $\quad+57-1-3208320 \quad$ (Ext 4140$) \quad$ (C.J.A-D.). aespejo@javeriana.edu.co; Tel.: +57-1-3208320 (Ext 4099) (A.J.E.M.)

\begin{abstract}
Sphingolipids are lipids derived from an 18-carbons unsaturated amino alcohol, the sphingosine. Ceramide, sphingomyelins, sphingosine-1-phosphates, gangliosides and globosides, are part of this group of lipids that participate in important cellular roles such as structural part of plasmatic and organelle membranes maintaining their function and integrity, cell signaling response, cell growth, cell cycle, cell death, inflammation, cell migration and differentiation, autophagy, angiogenesis, immune system. The metabolism of these lipids involves a broad and complex network of reactions that convert one lipid into others through different specialized enzymes. Impairment of sphingolipids metabolism has been associated with several disorders, from several lysosomal storage diseases, known as sphingolipidoses, to polygenic diseases such as diabetes and Parkinson and Alzheimer diseases. Sphingolipids are mainly located in central nervous system, and their abundance and distribution depend on brain development state and cell type. Although several studies have expanded the knowledge about sphingolipids function both in health and disease, it is still necessary to continue their study to understand the cellular implications of novel sphingolipids. These studies will also contribute to the diagnosis and treatment of diseases in which these molecules are part of their pathophysiology. In this review, we summarize the main sphingolipid characteristics and current knowledge about the synthesis, catabolism, regulatory pathways, participation in action potential and ionic channels, among other relevant functions.
\end{abstract}

Keywords: Central Nervous System, GM2 Gangliosidoses, Sphingolipids, Sphingolipidosis.

\section{Introduction.}

Sphingolipids are important structural constituents of cell membrane that participate as signaling molecules in regulatory pathways [1-4]. They are complex phospholipids formed by a sphingosine head and a long fatty acid chain, which could be classified as sphingomyelins (ceramide plus phosphocoline or phosphoethanolamine) and glycosphingolipids (cerebrosides, sulfatides, globosides, and gangliosides) [1, 5].

Sphingolipids are synthesized through a de novo pathway that begins in the endoplasmic reticulum, and are later modified into the Golgi apparatus and transported to the cell membrane 
through vesicular transport $[1,2,5]$. On the other hand, their catabolism involve several lysosomal enzymes [6]. Although the sphingolipids are widely distributed in different tissues as part of membranes or vesicles, glycosphingolipids and gangliosides are mainly located in the central nervous system (CNS), where represent almost $20 \%$ of the total lipids [7]. In this tissue, sphingolipids participate in physiological and neurodevelopmental processes providing adequate CNS function [3, 8].

It has been described that ganglioside expression change throughout brain development as well as in different brain regions and CNS cell types $[4,9,10]$. For instance, galactolipids and its sulfated derivate sulfatide are found in oligodendrocytes, glia cells and myelin; while gangliosides are mainly located in neurons (axon membrane) and grey matter [11]. Concerning to brain development, at embryonic state gangliosides molecules are simple, but due to formation of neural circuits during neural development, their structures change becoming more complex, which seem to remain in the adulthood $[8,11]$.

The sphingolipid heterogeneity and its distribution along the membrane of neural cells generate conformational changes and microdomains that participate in the specialized functions of polarized cells as neurons and oligodendrocytes [12-15]. Likewise, gangliosides are related to axonal growth, dendritogenesis, myelination, and modulation and stabilization of ion channels [16-20]. Thus, sphingolipids must play an important role in the establishment of synaptic relationships and connections between neurons, demonstrating their participation in neuronal processes to keep neuroplasticity and stimulate different contexts or types of memory and neural learning $[8,21]$.

Considering the different roles of sphingolipids into the brain's cells structure and development, is important to note that the impairment of their metabolism may result in cellular changes leading to different metabolic, neuronal, or proliferative disorders. The group of disorders caused by the disfunction of biosynthesis or catabolism of glycosphingolipids are known as sphingolipidoses [22], mainly caused by impaired lysosomal enzymes or by altered transport of cholesterol in the lysosomecytosol pathway. Due to the broad heterogeneity of this group, it was recently divided into genetic disorders of biosynthesis, defects of degradation, and diseases of lysosomal deposit [22, 23]. All of them can course from asymptomatic presentations to severe neonatal forms, and show organomegaly, bone abnormalities, and CNS progressive impairment conducting to neuronal degeneration $[24,25]$. Additionally, it has been described the impact of sphingolipids in other diseases such as cancer, diabetes, and cardiac disorders, among others.

Even though many characteristics and roles of sphingolipids have been described, there are some mechanisms that are not well understood. For this reason, it is important to continue addressing a greater understanding of the synthesis and degradation processes of these compounds, to understand the basis of some neurological disorders and possibly develop new therapeutic alternatives for these disorders. In this review, we provide a compilation of recent information about sphingolipid characteristics, brain distribution and their role in metabolism, neural development, ionic flux and their implications in several disorder

\section{Sphingolipids: Structure, metabolism, and central nervous system distribution.}

Sphingolipids are complex lipids composed of 18 carbon amino-alcohol backbone representing near $20 \%$ of the lipids in the nervous system which acts mainly as structural molecules of the cell membrane. They also participate in essential functions such as brain development, cell recognition, adhesion, and toxin receptors [1-4]. De novo biosynthesis of sphingolipids is a compartmentalized and fine regulated process that begins at the cytosolic leaflet of the endoplasmic reticulum (ER) from non-sphingolipid precursors to synthesize ceramide, in a pathway that involves enzymatic regulated steps in which the serine palmitoyltransferase is the key enzyme $[1,26]$. In the luminal face of the ER, this ceramide is modified through the addition of one galactose residue by the ceramide galactosyltransferase to form galactosylceramide (GalCer) and later transported to the Golgi apparatus [5]. The ceramide can also be directly transported from ER to the trans-Golgi network by the ceramide-transfer protein (CERT), a protein regulated by phosphorylation in response to cell 
sphingomyelin requirements $[1,27,28]$. In this later transport, the sphingomyelin synthase 1 catalyzes the ceramide conversion and phosphatidylcholine into sphingomyelin (SM) [27].

An alternative ceramide transport pathway to cis-Golgi from ER through coatomers and vesicular transport has also been described, although its regulation mechanism is not entirely understood [3]. At the cis-Golgi, the glucosylceramide synthase transfers glucose from UDP-glucose to the ceramide to form glucosylceramide (GlcCer). Then, the GlcCer is transported to the trans-Golgi network by the four-phosphate adaptor protein 2 (FAPP2) [29] to form different glycosphingolipids (GSL) $[3,28,30]$. In the brain, the UDP-Gal:GlcCer $\beta 1-4$ galactosyltransferase transfers a galactose unit to the GlcCer to form lactosylceramide (LacCer) which is modified by the addition of sialic acid by the LacCer- $\alpha-2-3$ sialyltransferase to form the simplest ganglioside GM3 at the trans-Golgi [7]. Several sugars attachment on the GM3 allows the synthesis of GM2 and GM1 gangliosides [7] to be finally released in Golgi vesicles to the lipids rafts at the plasma membrane, especially at synaptic surfaces [6], in a fusion membrane-dependent mechanism [31]. Figure 1 summarizes the sphingolipids biosynthetic pathway. GM1 and the GSLs GD1a, GD1b, GT1b, and GQ1b constitute up to $95 \%$ of all gangliosides in the brain $[7,31]$ and represent $20-25 \%$ of the outer leaflet of neuron membranes [32]. Although these chemical reactions are usually limited to the membrane organelles, the sialyltransferase has been found as membrane-associated enzymes suggesting pivotal roles in the GSL remodeling [33-35].

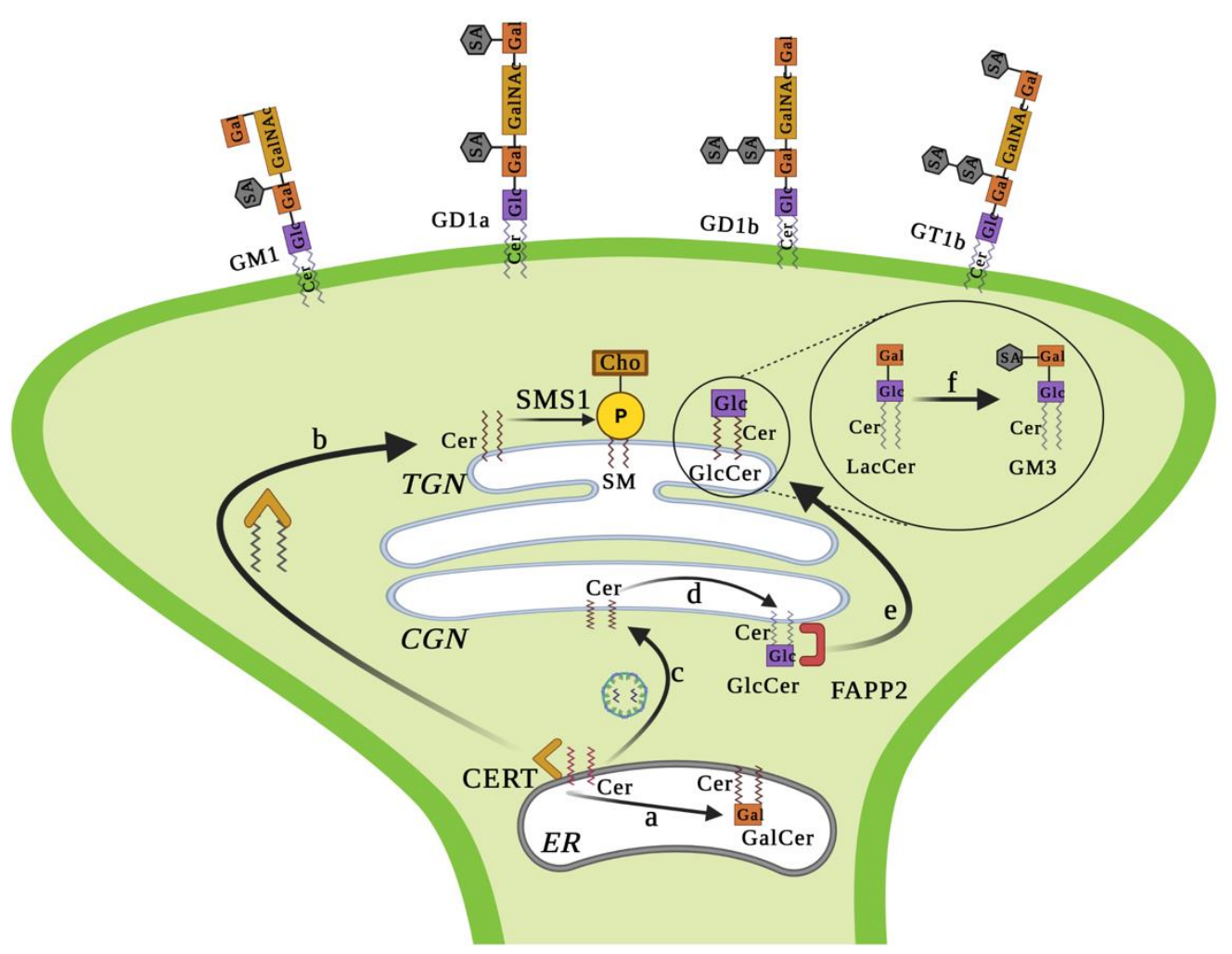

Figure 1. de Novo biosynthetic pathway of sphingolipids. Ceramide (Cer) synthesis is the first step in the synthesis of the sphingolipids which is carried out in the cytosolic leaflet of the endoplasmic reticulum (ER). Upon its synthesis, the ceramide is modified by the addition of galactose (a) forming GalCer into the ER lumen, since where it seems to reach the cis-Golgi network (CGN). The ceramide can be transported from ER to the trans-Golgi network in an independent vesicular transport by the ceramide-transfer protein (CERT) (b), where the sphingomyelin synthase 1 (SMS1) catalyzes the synthesis of sphingomyelin (SM). On the other hand, the ceramide can be coated and transported to the CGN since ER (c). Once there, the glucosylceramide synthase adds glucose to the ceramide to form GlcCer (d). This GlcCer can be transported to the TGN by the fourphosphate adaptor protein 2 (FAPP2) (e). The addition of the galactose and the further sialic acid allows the synthesis of the simplest ganglioside GM3 (f) which is the precursor of major gangliosides. With the GM1 
ganglioside, the GD1a, GD1b, GT1b are the most abundant sphingolipids in the brain. This figure was created with BioRender.com.

While the biosynthetic route involves the secretory pathway, the catabolism of sphingolipids requires the endocytosis and the activity of several lysosomal enzymes [6]. Besides, catabolic enzymes like neuraminidase [36], hexosaminidase [37], and galactosidase [38] were early found at the plasma membrane. Respect this, early studies performed by Rodriguez et al., showed that plasma membrane neuraminidase contributes to the regulation of axonal growth and regeneration of hippocampal neurons using in vitro approaches [39], suggesting a fine regulation of GSL in the central nervous system. These interesting topics will be discussed later.

\subsection{Sphingolipids profile in the Central Nervous System}

Even though the sphingolipids are virtually distributed throughout all the brain, differential profiles have been described during the embryonic period, childhood, and adulthood, as well as in different brain regions and CNS cell types $[3,8]$. In this respect, it was reported that in rat brains the overall ceramide species are increased since post-natal day 1 (P1) up to P21 and then they are reduced from P30 to adult. This behaviour is believed to stimulate the biosynthesis of several sphingolipids such as the GM1 ganglioside [4]. In this scenario, recently in adult rats, it described the presence of complex GSL such as sulfatides, LacCer, and hexosylceramide in the dorsal hippocampus, a crucial brain structure related to learning, memory, and emotional responses. Enrichment of short ceramides (C16-C20) accompanied by reduced levels of longer ceramide (C22-C26) have been found in the ventral hippocampus [40]. In contrast with this physiological profile, the corticosterone-induced stress in adult rats has shown an increase in the dorsal-ventral sphingolipid catabolism, suggesting that the stress could trigger CNS diseases. Besides, sphingolipid changes have been reported in the brain tissues isolated from autist patients [41].

GD1a, GD1b, GM1, and GT1b are the major gangliosides in the adult mammalian brain [8] and similar findings have also been reported in human brains (Figure 2) [11, 42, 43]. Consistent with the above, the expression of some galactolipids also changes during murine brain development. In this line, the GM3, GM1, GD1a, GD1b, GT1b, GM2, GD3 gangliosides are enriched in the early developmental stages (primary and secondary follicle), whereas in the blastocyst the GM3 and GT1b are predominant during the mouse embryogenesis [9]. At the embryonary stage ( E15) as well as the P2-P5, the GlcCer is the only monoglycosylceramide present, whereas at P8-P10 begins the synthesis of GalCer which increase up to P21-P25 [4, 10]. These findings suggest that murine brain development is an age-dependent dynamic process, in which sphingolipids seem to be pivotal regulators. Decreased levels of GalCer are frequently found in multiple sclerosis rat brains [4], an autoimmunedependent demyelinating disease. 


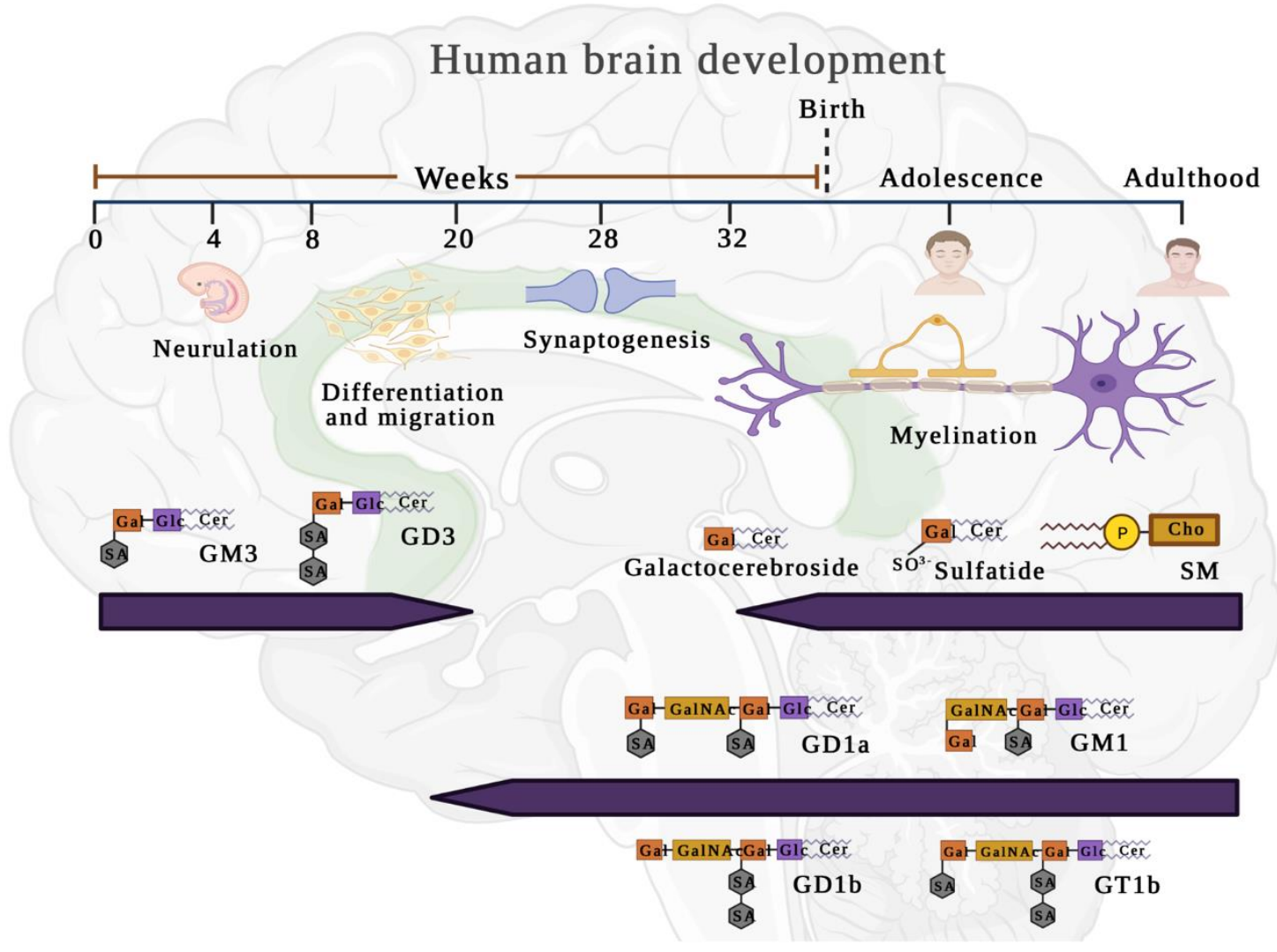

Figure 2. Overall Sphingolipid profile during the human brain development. Note that during the early embryonic stages there are predominantly simple gangliosides, which change to most complex structures in consequence of the neural circuit's formation. This later profile of sphingolipids seems to remain in adulthood. This figure was created with BioRender.com.

As expected, changes in the sphingolipid's arrangement are also found at the different CNS cell types (Figure 3). For instance, neurons are enriched in gangliosides (up to $12 \%$ of total lipid content) at the axonal membrane by GD1a and GT1b forming microdomains that promote myelin stabilization through the interaction with the myelin-associated glycoprotein (MAG) [13, 44]. Although GD3 synthesis increase during the early neurogenesis, this is not able to bind MAG, suggesting that GD3 is a key start point to more complex gangliosides without direct interaction with MAG [44]. Concerning glia cells, it is well known that the oligodendrocytes plasma membrane is enriched with GalCer and sulfatide [14]. A recent study provided an interesting finding in P16 rat-derived oligodendrocytes where GlcCer and sphingomyelins represented the major ceramide species [15]. Despite the above, it is clear that myelin lacks GlcCer, and even blocking the biosynthesis of GlcCerderived sphingolipids does not result in aberrant myelinization in the CNS [45]. Therefore, the function of this pool of GlcCer in oligodendrocytes remains to be elucidated. Although myelinization in the CNS is driven by the oligodendrocytes, previous reports have suggested that this process seems to depend on the astrocyte-derived lipids rather than from oligodendrocyte lipid synthesis [46]. In astrocytes, in addition to GM3 [47], high amounts of ceramide and sphingosine 1P (S1P) derived from sphingosine metabolism have been found [48, 49], which are well-established as bioactive compounds in health and disease [50-52]. Finally, in microglia different sphingolipids patterns can be observed according to its activation stage. In this aspect, proinflammatory roles have been associated with long-chain ceramides (C16-C24) and S1P, whereas short-chain ceramides exert antiinflammatory effects in the CNS [53-55]. 

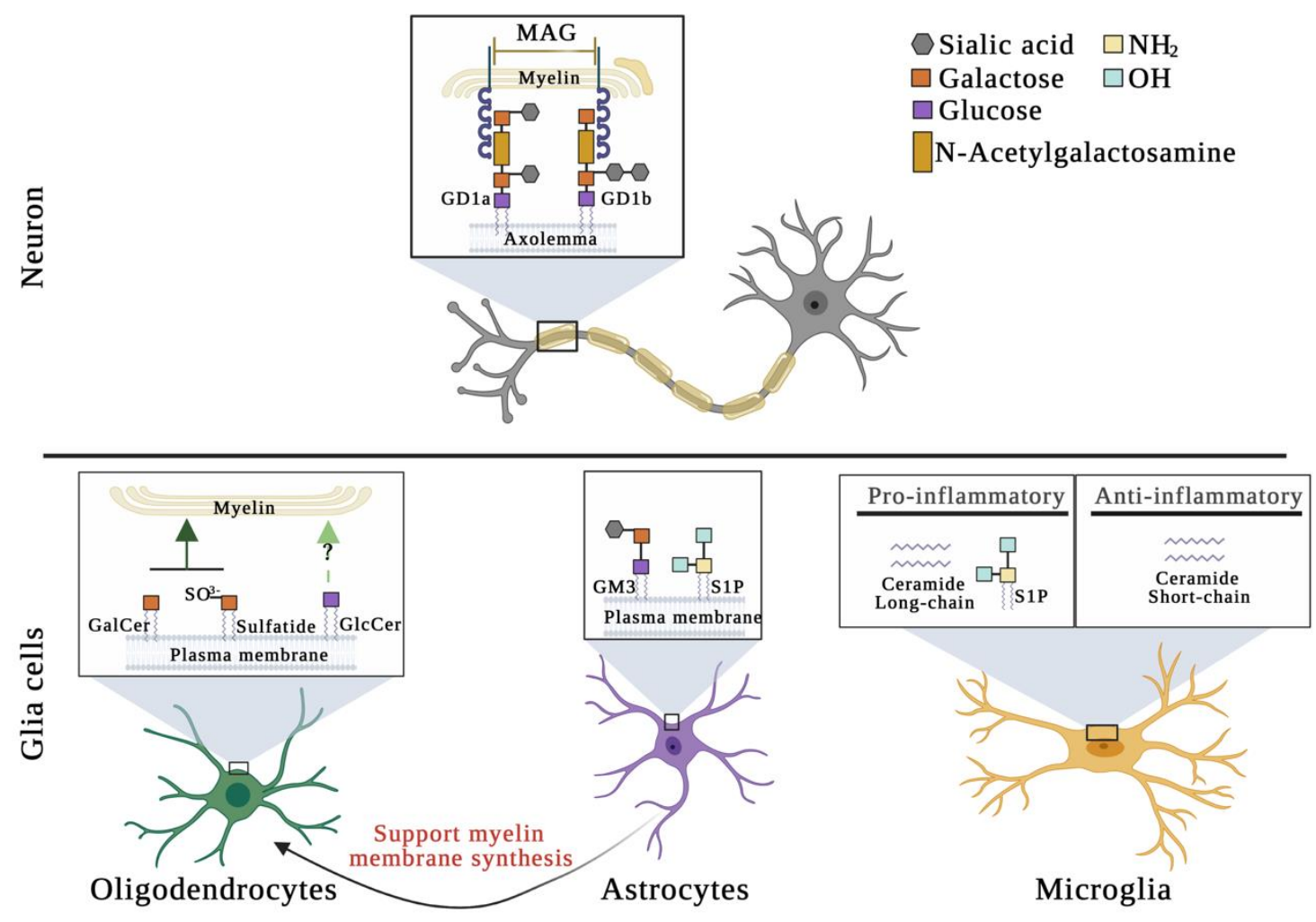

Figure 3. Major sphingolipids in the CNS cells. Complex sphingolipids are present in neuron's axon plasma membrane, which interact with the myelin-associated glycoprotein (MAG), contributing to the myelin stabilization. The GalCer and sulfatides are found in the oligodendrocytes; however, high amounts of GlcCer have also been described [45]. Nevertheless, its role in the myelinating oligodendrocytes has not to be elucidated. Although traditionally it has been accepted that the lipid synthesis by oligodendrocytes is crucial to the myelination of the axon, recently it was established that the astrocytes support this process through the cholesterol and unsaturated fatty acids synthesis [46]. Finally, the pro-inflammatory (M1) and anti-inflammatory (M2) microglia stages are accompanied by different sphingolipids profiles where the ceramide length seems to be crucial for the differential signalling in these immune cells. This figure was created with BioRender.com.

\section{Sphingolipids-mediated cellular signaling pathways.}

Neurons and oligodendrocytes are highly polarized cells with particular morphology that allow them to carry specialized functions [8]. The particular heterogeneity in sphingolipid (SL) distribution along the cellular membrane of these cells produces microdomain conformations that play important roles in neuronal differentiation, synapse formation, glial-neural interaction, and polarization [8]. In this section, we review the current knowledge about the functions described to the sphingolipids at the CNS, which are summarized in Figure 4. 


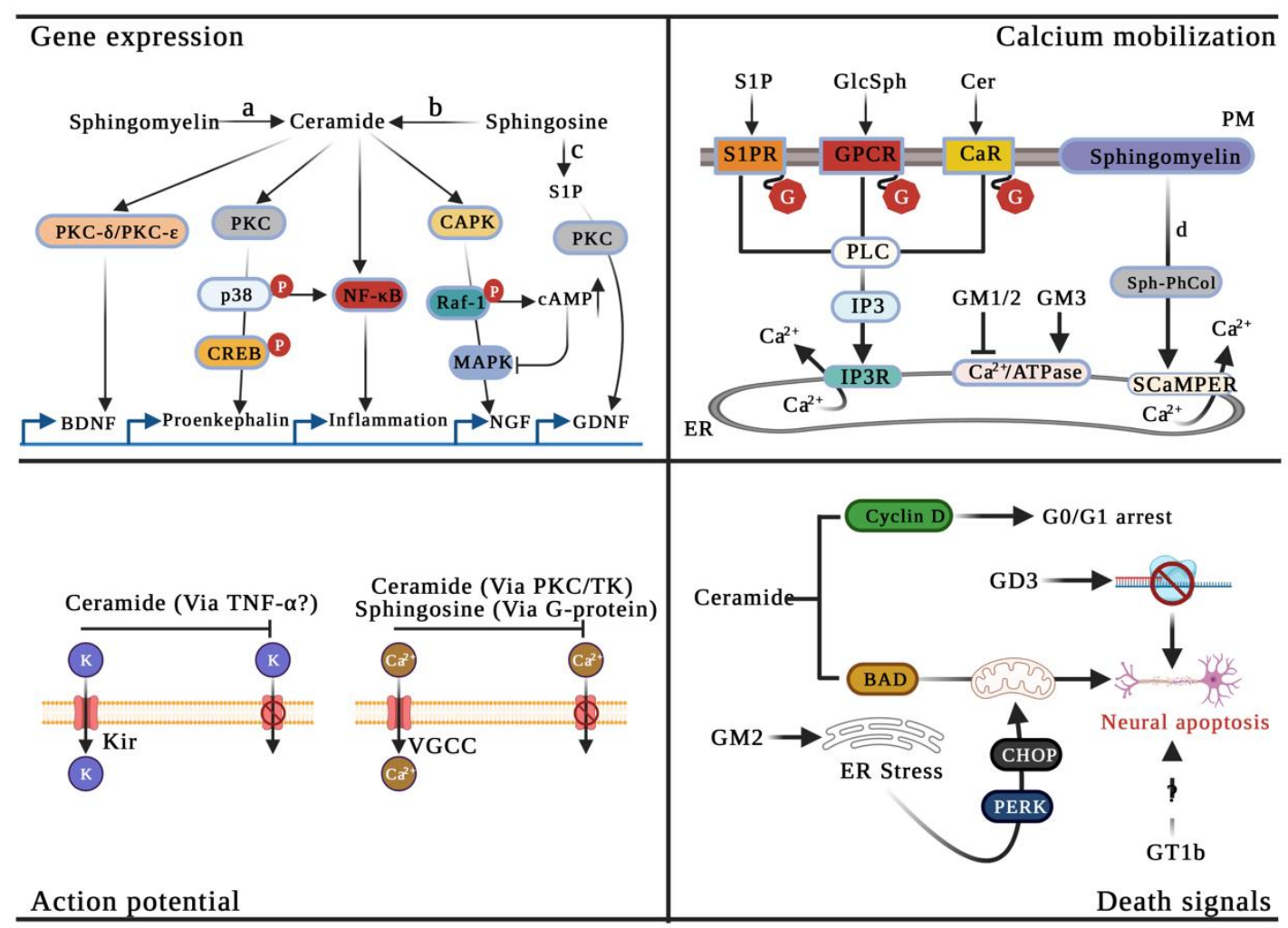

Figure 4. Role of sphingolipids in cell signaling. Gene expression: Ceramide and Sphingosine-1phosphate (S1P) can influence the actions of several signal effectors such as $\mathrm{CAPK}, \mathrm{nF} \kappa \mathrm{B}, \mathrm{PKC}$, and its isoforms $\mathrm{PKC} / \mathrm{PKC} \varepsilon$, to promote the gene expression of some proteins such as BDNF, proenkephalin, NGF, and GDNF. Also, inflammation can be induced by overexpression of different proteins (refer to the text for more details). (a) the enzymatic reaction catalyzed by sphingomyelin phosphodiesterase. (b) the enzymatic reaction catalyzed by sphingosine ceramide synthase. (c) the enzymatic reaction catalyzed by sphingosine kinase 1 and 2. Calcium mobilization: SP1, Glucosyl sphingosine (GlcSph), and ceramide (Cer) can activate G protein-coupled receptors to promote calcium release via IP3R activation. GM1/2 and GM3 can both inhibit or activate the endoplasmic reticulum calcium pump $\mathrm{Ca}^{2+} / \mathrm{ATPase}$, respectively. Sphingosyl phosphorylcholine (Sph-PhCol) acts over the SCaMPER channel and promotes calcium release. PM: Plasma membrane. ER: Endoplasmic reticulum. (d) the enzymatic reaction catalyzed by sphingomyelin deacetylase. Action potential: Ceramide induces a close state in Inwardly Rectifying Potassium (Kir) Channels probably via TNF- $\alpha$. Also, ceramide and sphingosine act over Voltage activated calcium channels (VGCC) inducing inhibition of these channel-mediated currents. Death signals: G0/G1 arrest and caspase-mediated cell death are induced by ceramide build-up. Cell death by GM2 and GT1b build-up can promote cell death by endoplasmic reticulum stress and mitochondria-dependent pathway, and an unknown mechanism, respectively. GD3 increase inhibits DNA synthesis. Although sphingolipids are involved in cell death signals, they also can promote cell survival through PKC, PKCठ/PKCE, $\mathrm{NF} \kappa \mathrm{B}$, and CAPK in the case of ceramide and IP3 dependent or independent mechanism in the case of Sphingosine-1-phosphate (not shown). This figure was created with BioRender.com.

\subsection{Sphingolipids in neural differentiation, dendritic formation axonal determination, and growth}

During embryonic development, the sphingolipid profile into the brain changes. The changes and distribution of some sphingolipids in membrane microdomains suggest that they are essential for some functions as modulation of signaling transduction, cell adhesion, and neural differentiation $[8,56]$. For instance, the GD3 ganglioside is highly expressed in the embryonic mouse brain (particularly in neuronal stem cells - NSC) and decreases dramatically after birth [57], suggesting an important role during embryonic development. In previous studies, it has been shown that GD3 interacts with the epidermal growth factor receptor to support the neural self-renewal of NSCs 
through the EGF-induced EGFR signaling [57]. GD3 synthase knockout in mice produces a decrease in NSCs populations proving its essential role in the sustaining of these cells [58].

On the other side, gangliosides have been linked to dendrite and axon growth [16]. In early reports, it was shown that GM2 ganglioside accumulation let abnormal neuronal geometry and induces ectopic dendrites in patients with Tay-Sachs disease, and cat models that mimic GM2 gangliosides [59, 60]. It is thought that GM1 ganglioside is involved in calcium flux between nucleus and cytoplasm through the stabilization of the $\mathrm{Na}^{+} / \mathrm{Ca}^{2+}$ exchanger in the inner membrane of the nuclear envelope during the neural differentiation [17], supporting the previous hypothesis. Likewise, the GD3, GD1b, and GT1b gangliosides are related with dendritogenesis by modulation of the calmodulin kinase II (CaM-KII) pathway in primary cultured neurons, supporting that ganglioside/CaM-KII could be a mechanism to dendritic morphogenesis of growing neurons [18]. This is consistent with early studies suggesting the need for sphingolipids biosynthesis for dendrite growth $[19,20]$.

Concerning axon growth, it has been observed that ceramide synthesis inhibition reduces the axonal length on cultured hippocampal neurons probably because sphingolipids are used as membrane molecules for axonal elongation [61]. Also, the polarization of neurons, which is triggered by the axon specification and requires the localized destabilization of filamentous actin, seems to be modulated by the G1-mediated downstream signaling in a plasma membrane ganglioside sialidasedependent mechanism [62]. A similar role has been associated with a GD3 derivative (9-Oacetyl GD3) [63].

\subsection{Sphingolipids' role in action potential and ionic channels}

Proper myelination is necessary to confer neurons electrical insulation and proper action potential propagation [64]. Due to action potentials are propagated in a saltatory manner from one node of Ranvier (spaces of the axon devoid of myelin) to another due to the opening of voltage-gated sodium channels. The myelination process involves interaction among several molecules like proteins, glycoproteins, and lipids [65]. During the myelination process, some sphingolipids participate in a complex fashion, although the exact mechanism in which they interact is largely unknown [4]. More specifically, Ceramide (Cer)/dihydroceramide (dhCer), Sphingosine (Sph)/dihydrosphingosine (dhSph), and glucosyl/galactosylceramide (Glc/GalCer) participate in myelin formation during mice brain development [4]. GalCer-enriched microdomains seem to colocalize with several proteins of the myelin sheaths and some examples of this are the interaction with myelin basic protein (MBP), 20, 30 -cyclicnucleotide 30-phosphodiesterase (CNP), myelin/oligodendrocyte glycoprotein (MOG), and proteolipid protein (PLP) [66]. Besides its interaction with different myelin proteins, GalCer is important for myelin stability since GalCer synthesis impairment has been associated with myelin degeneration and encephalopathy $[67,68]$. The GM2/GD2 are also important for normal microdomain composition in nodes of Ranvier since the abnormal synthesis of these gangliosides alters its typical microdomain composition as well as the normal $\mathrm{K}^{+}$and $\mathrm{Na}^{+}$ion channels localization $[69,70]$.

Synapses are structurally complex and required regulated compartmentalization of membrane components to maintain critical functions such as neural plasticity that is defined as the brain's ability to adapt to different stimuli and undergo learning memory processes and behavior changes $[8,71]$. In this context, sphingolipids play a transcendental role as several studies have shown that the balance between SM and ceramide in the hippocampus is associated with post-synaptic excitatory transmission, which is pivotal to spatial memory and episodic-like memory [21, 72]. The so-called Long-Term Potentiation (LTP) is another key phenomenon occurring in neurons of the central nervous system that is associated with the memory and learning process [8]. Among the several mechanisms governing LTP, the proper clustering of glutamate receptor, N-methyl-D-aspartate receptors (NMDARs) seems to be related to sphingolipids microdomain, suggesting that these molecules play an important role in NMDA mediated LTP [71, 73]. Besides, another glutamate receptor ( $\alpha$-amino-3-hydroxy-5-methyl-4-isoxazole propionic acid, also known as AMPAR) mediates 
fast synaptic transmission, and its trafficking is a critical component of learning and memory [74]. Prendergast and colleagues report a model in which GluR2 subunits of AMPAR complex and an AMPAR trafficking complex containing Nicalin, Thorase, and NSF are proposed to be sequestered in distinct membrane rafts by association with gangliosides GM1 and GT1b, respectively. In an ATPdepending process, the ganglioside interactions are released, resulting in lateral migration, the association of AMPA receptors with their trafficking complexes, and receptor endocytosis [75].

Regarding ion channels, a great number are found associated with microdomains, although how sphingolipids influence their organization and function are only well defined for some cases [8]. For instance, SM has been shown to influence Kv2. 1, Kv1.3, and Kir1.1 channel activity by interacting with S3b and S4 voltage-sensing domains with positively charged residues through interaction with phospho-heads of membrane lipids to produce energetic stabilization [16, 76]. The overall effect of the hydrolysis of SM into ceramide is the inhibition of $\mathrm{K}+$ conductance and hyperpolarization shift in the voltage-conductance relation for Kv 1.5 and Kv2.1 [16, 76]. Microdomains mediate ion channels and kinase proteins co-localization, which is important since phosphorylation is a mechanism of ion channel regulation [8]. It has been proved that phosphorylation suppresses depolarization-evoked currents and disruption of microdomains by cholesterol depletion and inhibition of sphingolipids synthesis produce a hyperpolarizing shift of Kv1.5 channels in Ltk cells [77]. Another mechanism by which sphingolipids may influence ion channel activity is through the regulation of scaffolding proteins as PSD-95, which has been proved to increase in the mouse brain when SM/ceramide balance is altered and is important for $\mathrm{Kv} 1.4$ and NMDA receptor recruitment into microdomains [21, 78].

Finally, it has been described that sphingolipids are involved in calcium mobilization [79]. Particularly, S1P and sphingosine induce $\mathrm{Ca}^{2+}$ via Inositol triphosphate (IP3) and PKC inhibition in the case of sphingosine and sphingosylphosphorylcholine (SPC) [80, 81]. A specific SPC-activated calcium channel called SCaMPER has been identified in the endoplasmic reticulum and is proposed to modulate calcium mobilization via SPC signaling [79, 81]. Evidence shows that sphingomyelinase increased activity by tumor necrosis factor- $\alpha$ (TNF $\alpha$ ) induction or elevated expression produce transient $\mathrm{Ca}^{2+}$ inward currents in neonatal rat dorsal root ganglion (DRG) and fura-2-loaded neurons [79]. In contrast, GlcCer and glucosylsphingosine have been reported to mobilize calcium via the ryanodine receptor [82]. The glucosylsphingosine seems to also regulate $\mathrm{Ca}^{2+}$ mobilization in an IP3 and $\mathrm{Ca}^{2+} /$ ATPase dependent manner $[82,83]$.

\subsection{Sphingolipids and signaling pathways in cell death and survival}

Sphingolipids, particularly ceramide, have an important role in cell death [79]. Ceramide can activate both intrinsic and extrinsic cell death pathways by interacting directly or not with different effectors such as Fas-L, TNF $\alpha$, NGF, MAPK (ERK, JNK, and p38), and Akt [79, 84]. Specifically, ceramide treatment dephosphorylates Akt, releases cytochrome $\mathrm{c}$, and produces caspase 3 release [85]. Akt inactivation is also seen in motor neurons [79]. Early studies have shown that ceramide induces reactive oxygen species (ROS) production by inhibiting the complex III of the respiratory chain [86-88]. There is evidence of a proapoptotic effect of the GD3, GT1b, and GM2. In the case of GM2, the accumulated ganglioside in GM2 gangliosidoses [7], it was recently shown that its accumulation induces PKR-like endoplasmic reticulum kinase (PERK)-mediated apoptosis in N2a cells [89]. In contrast with these proapoptotic sphingolipids, the GM1 ganglioside seems to have a neuroprotective effect on glutamate toxicity through the activation of the Trk receptor [90].

Some sphingolipids can regulate cell proliferation and survival [79]. For instance, evidence suggests that sphingosine kinase1/S1P signaling promotes the proliferation and survival of neurons in a PI3K/Akt-mediated pathway, supporting a key role in neurogenesis [91, 92].

\section{Impaired sphingolipids metabolism on the central nervous system.}


The diseases that compromise the biosynthesis or catabolism of glycosphingolipids are known as sphingolipidoses. Although the pathogenesis of these diseases is not completely understood, several approaches have been carried out in order to develop treatment strategies and allow a specific and early diagnosis. Figure 5 shows an overview of the significant pathways of sphingolipid catabolism and the related sphingolipidosis.

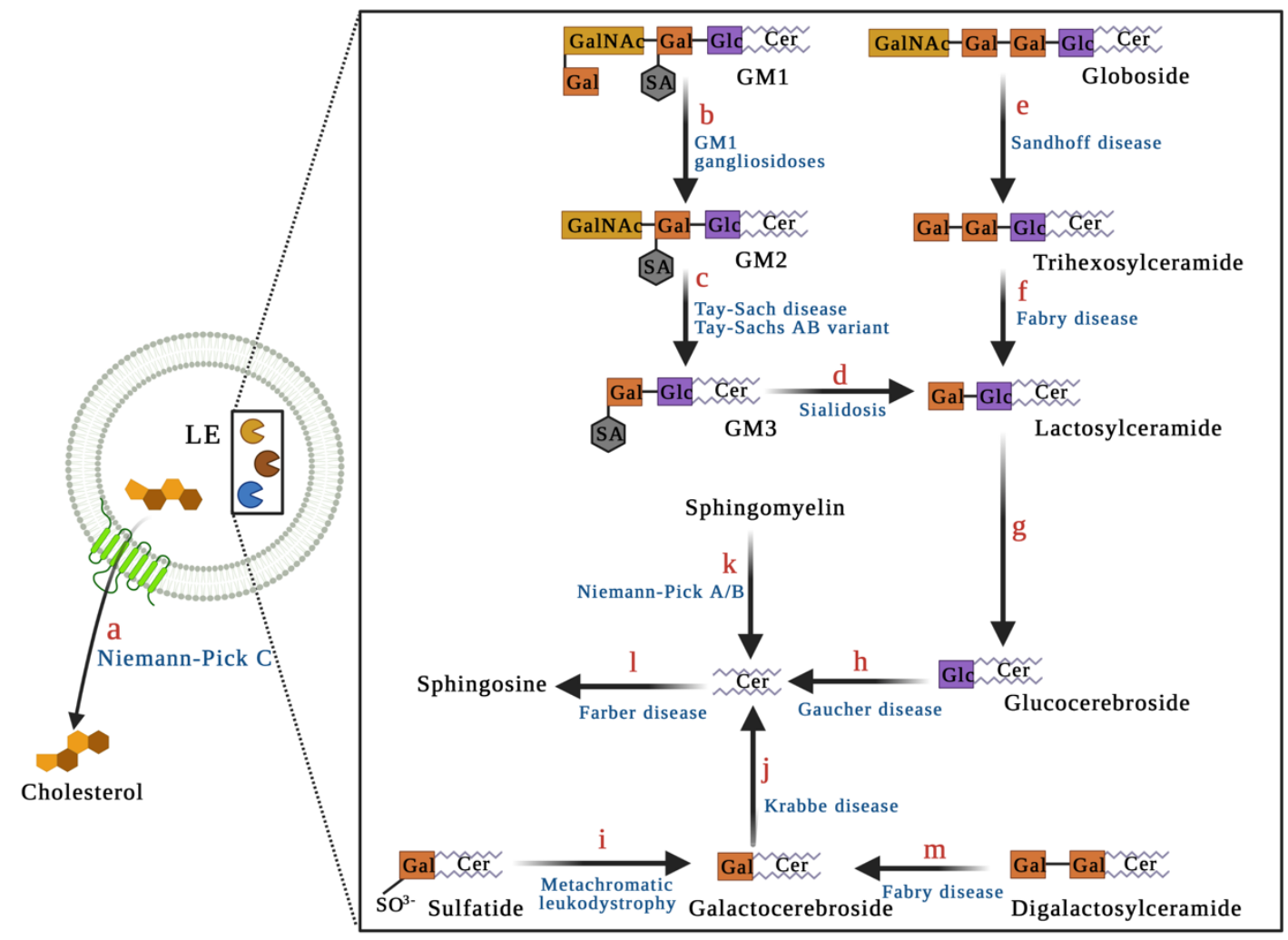

Figure 5. Sphingolipid catabolism and sphingolipidosis. Although most sphingolipidoses are caused by the impaired lysosomal enzyme (LE) activity, Niemann-Pick Type C (NPC) is originated from the altered transport of cholesterol in the lysosome-cytosol pathway allowing its accumulation into the lysosome and affecting the lysosomal function (a). In the case of the ganglioside catabolism during the endocytosis and membrane renewal membrane, some species as GM1 are degraded to GM2 by the remotion of Gal in a catabolic step catalyzed by the galactosidase $\beta-1(\mathbf{b})$. Upon the resulting GM2 ganglioside is removed a unit of GalNAc by the hexosaminidase A in a GM2 activator protein-dependent mechanism (c). The further remotion of the SA in the GM3 ganglioside by the neuraminidase/protective protein/cathepsin A-PPCA (d) allows the formation of the lactosylceramide. On the other hand, the globoside, which differs from the GM1 ganglioside only by the presence of the SA in the latest can be catalyzed by the hexosaminidase B with no participation of the GM2 activator protein (e). The resulting trihexosylceramide is subject to the enzyme activity of the galactosidase alfa/Saposin B (f). In both cases, the degradation of the GM1 ganglioside and the globoside allows the formation of the lactosylceramide which lost a Gal by the action of the galactosidase/Saposin B and C (g) to form glucocerebroside. This simple glycolipid is subject to the catalysis of the glucosylceramidase beta/Saposin C (h) to obtain ceramide. In a parallel catabolic pathway, the sulfatides can form galactocerebrosides by the action of the arylsulfatase A/Saposin B (i). The galactocerebroside is additionally catalyzed by the galactosylceramidase/Saposin A and C (j) allowing the formation of ceramide. Likewise, the enzyme activity of the sphingomyelin phosphodiesterase $1 /$ acid ceramidase on the sphingomyelin (k) from ceramide too. This ceramide is a potent pro-apoptotic molecule by which it must be transformed to sphingosine by the action of the ceramidase/Saposin D (1). An alternative catabolic pathway where the digalactosylceramide is catabolized by the galactosidase alfa/Saposin B ( $\mathbf{m})$ to galactocerebroside is shown also. The impaired enzymatic activity of any of these enzymes can produce several sphingolipidoses which are shown in light blue font. Whereas Tay-Sachs disease corresponds to hexosaminidase A deficiency, the Tay-Sachs AB variant refers to the deficiency of the GM2 activator protein. This figure was created with BioRender.com. 
Currently, it has been described more than 70 lysosomal diseases related to the catabolism of several substrates, including the sphingolipids, which are accumulated into the lysosome as a consequence of the mutations on the gene encoding to proteins related to the lysosomal function [22]. An interesting review made by Aerts et al., 2019 summarized the main findings of sphingolipidosis using Gaucher disease as a model [25].

In 2020 Pakanova et al. expand the concept of sphingolipidosis using the term glycoconjugate metabolism disorders, including glycan biosynthesis disorders, glycoconjugate catabolism, congenital glycosylation diseases, and lysosomal storage diseases [23]. Ryckman et al., 2020, reorganizes the division of glycosphingolipidoses into genetic disorders of biosynthesis, defects of degradation, and diseases of lysosomal deposit, very similar to the initial classification proposed by Platt et al., in 2014 [22, 93]. Table 1 summarizes the current classification of main diseases that affect sphingolipid metabolism and their main characteristics.

Regardless of the classification, sphingolipidoses are characterized by extensive clinical heterogeneity, mainly caused by the accumulation of partially degraded substrates, which can be observed from asymptomatic presentations to severe neonatal forms. It is common to find organomegaly, bone abnormalities, and CNS disorders that usually have a typically degenerative presentation [23,24]. The sphingolipids can start at very early stages of life, without meaning that they cannot observe later presentations, equally with varying degrees of severity [22]. According to clinical diversity, the diagnostic approach for these disorders is equally controversial and different diagnosis alternatives can be performed from neonatal screening to allow early therapeutic interventions, when these are available, as well as biomarkers identification. In this last point, significant advances have been done using mass spectrometry technology [94, 95], as well as in the improvement of enzyme assays on different biological samples (urine, plasma, saliva, leukocytes, fibroblasts) or dry blood spots on filter paper, and molecular tests that in some cases allow to confirm the diagnostic $[96,97]$. 


\begin{tabular}{|c|c|c|c|c|}
\hline Group & Disorder Name & $\begin{array}{c}\text { Protein } \\
\text { Affected }\end{array}$ & $\begin{array}{l}\text { OMIM } \\
\text { number }\end{array}$ & Main Clinical Features \\
\hline \multirow{3}{*}{ AD } & GM2 activator & GM2 activator & 272750 & Like to GM2 gangliosidosis. Epileptic fits, macular cherry -spot, muscle weakness \\
\hline & Prosaposin & Sap precursor & 176801 & \multirow{2}{*}{ Clinical features are often indistinguishable from the corresponding enzyme defect } \\
\hline & Saposin disorders & Sap A to Sap D & 611721 & \\
\hline N/A & Farber Disease & Acid ceramidase & 228000 & Macular cherry-spot, progressive neurologic disability, hepatosplenomegaly \\
\hline NPD & Type A, B, and C & $\begin{array}{l}\text { AS (A and B) } \\
\text { and LTD (C) }\end{array}$ & 257200 & $\begin{array}{l}\text { Hepatosplenomegaly, progressive pulmonary infiltration, failure to thrive, progressive } \\
\text { neurodegenerative course }\end{array}$ \\
\hline GD & Type 1,2 and 3 & A $\beta$ Glu & 231000 & Hepatosplenomegaly, severe bone compromise, hematological signs \\
\hline N/A & Krabbe Disease & Gal & 245200 & Motor deterioration, Cognitive decline, corticospinal tract signs \\
\hline N/A & ML & ASA & 250100 & Gross motor deterioration, progressive nervous system compromise \\
\hline \multirow[b]{2}{*}{ Gangl. } & GM1 & $\beta$-Gal & 230600 & Progressive neurological deterioration, generalized rigospasticity, sensorimotor \\
\hline & GM2 & Hexo & $\begin{array}{l}272800 \\
268800\end{array}$ & Seizures, axial hypotonia, intellectual disability, motor regression, psychotic episodes. \\
\hline N/A & Fabry Disease & $\alpha$-Gal & 301500 & Pain and paresthesias in the extremities, hypohidrosis, corneal opacities \\
\hline N/A & Schindler Disease & NAG & 609241 & Neuroaxonal dystrophy, cortical blindness, myoclonus, seizures \\
\hline \multirow{4}{*}{ GDD } & $\alpha$-Mannosidosis & $\alpha$-Man & 248500 & progressive mental retardation, hepatosplenomegaly, severe dysostosis multiplex \\
\hline & $\beta$-Mannosidosis & $\beta$-Man & 248510 & Status epilepticus, severe quadriplegia, angiokeratomas \\
\hline & Fucosidosis & $\alpha$-Fuc & 230000 & Psychomotor retardation, coarse facies, growth retardation, dysostosis multiplex \\
\hline & Sialidosis & Neu & 256550 & $\begin{array}{l}\text { Mucopolysaccharidosis-like phenotype with visceromegaly, dysostosis multiplex, and } \\
\text { mental retardation. }\end{array}$ \\
\hline
\end{tabular}

Table 1. Main Sphingolipid Disorders Modified from: The Online Metabolic and Molecular Bases of Inherited Disease. 2019 [24]. AD: Activator Defects. NPD: Niemann-Pick Disease. GD: Gaucher Disease. Gangl: Gangliosidoses. GDD: Glycoprotein degradation disorders. N/A: Non-applicable. AS: Acid Sphingomyelinase. ML: Metachromatic Leukodystrophy. LTD: Lipid trafficking disorder. NAG: $\alpha$-N-Acetylgalactosaminidase. A $\beta$ Glu:-Acid $\beta$-glucosidase. Gal: Galactocerebrosidase. ASA: Arylsulphatase A. $\beta$-Gal: Beta-galactosidase. Hexo: Hexosaminidases. $\alpha$-Gal: $\alpha$-Galactosidase. $\alpha$-Man: $\alpha$-Mannosidase. $\beta$-Man: $\beta$-Mannosidase. $\alpha$-Fuc: $\alpha$-fucosidase. Neu: Neuraminidase 


\section{GM2 gangliosidoses as a model of sphingolipidosis}

GM2 gangliosidoses are a close group of sphingolipidosis characterized by the disfunction of the activity of $\beta$-hexosaminidases or the GM2 activator protein. The pathology leads to the accumulation, mainly in the neurons, of the GM2 ganglioside with a progressive neuron loss and the subsequent CNS impairment [7].

Since gangliosides are pivotal for membrane structure and critical functions in several CNS cells. Nevertheless, our understanding of the disturbance in the signaling pathway during the GM2 gangliosidoses is limited. In this context, Virgolini et al., recently showed that the GM2 ganglioside overload in neurites activates the mitochondrial-dependent apoptosis in a PERK-mediated mechanism, suggesting prolonged endoplasmic reticulum stress [89]. These findings agree with early reports using microsomes isolated from a Sandhoff disease mouse model (HEXB-/-) where a reduction in the $\mathrm{Ca}^{2+}$-uptake via sarco-endoplasmic reticulum $\mathrm{Ca}^{2+}-\mathrm{ATPase}$ was observed. This reduction was prevented after the pharmacological inhibition of the ganglioside synthesis by the Nbutyldeoxynojirimycin [98]. The molecular mechanisms of ER stress seem to be dependent on the presence of sialic acid in the GM2 ganglioside structure $[89,98,99]$. Further modulators of the PERKmediated apoptosis pathway could improve the prognostic of this sphingolipidosis in combined therapies. Consequently, several pro-inflammatory cascades can be activated in both innate and acquired immune responses [7]. For instance, microgliosis and infiltration have been observed in GM2 gangliosidoses patients and animal models [100-102]. In this scenario, previous reports of the neurodegenerative progress in a Sandhoff disease mouse model showed that there is an increase in the immunoreactivity for the Mayor Histocompatibility Complex type II in the brain stem and thalamus and an increase in the proinflammatory cytokines TNF $\alpha$ and IL1 $\beta$ [103]. Astrogliosis has also been reported in GM2 gangliosidoses because of the GM2 ganglioside accumulation, whit an increase in the secretion of the chemokines CCL2 and CXCL10 [104, 105]. Activated astrocytes may participate in degeneration and death of myelinating oligodendrocytes, promoting active demyelination [106, 107]. In this sense, these findings show the key role of GM2 ganglioside in brain inflammatory process, development, and immunomodulation.

Finally, it was recently reported the association of $\alpha$-synuclein with lipids (gangliosides) in rat dopaminergic neurons, which was prevented through the overexpression $\beta$-hexosaminidases [108]. The authors suggested that the interaction between lipids and $\alpha$-synuclein may occur in synaptic and vesicular domains due to the presence of gangliosides in lipid rafts located in these domains. These results correlate with previous findings showing that $\beta$-hexosaminidases deficiency induce $\alpha$ synuclein accumulation in a Sandhoff disease mouse model $[109,110]$. In addition, the loss of the ability to catabolize GM2 gangliosides led to the formation of high molecular weight $\alpha$-synuclein aggregates into the brain, as well as an increase in polyubiquitin [108], which suggest that GM2 gangliosides metabolism affects the function of the ubiquitin-proteosome and autophagy-lysosome pathways. These results shown the role of gangliosides in cell homeostasis and may contribute to the understanding of the physiopathology of several CNS disorders.

\section{Conclusions and Perspectives}

In this review we have described several aspects related to the synthesis, metabolism, and function of sphingolipids. These bioactive lipids are non-structural and non-energetic lipids that participate on cell homeostasis as well as in health and disease. Although they have been studied since late $19^{\text {th }}$ century, the role of most of sphingolipids are not completely understood, and novel structures and modifications are still identified, which shows the importance to continue the identification and characterization of these group of lipids. For instance, it was recently demonstrated that in addition to serine and palmitate, other substrates, such as alanine, glycine, myristate and stearate, can also enter sphingolipid metabolism, and generate novel lipids with potential previously no described cellular functions [111-113]. The synthesis and catabolism of these lipids are carried out 
through a highly regulated network of enzymes and transporters, for which their characterization has shed light not only on sphingolipids metabolism but also on most of the cellular regulatory networks. In addition, the presence of tissue-, organelle-, and substrate-specific isoforms of these enzymes adds an extra layer of complexity to the sphingolipid metabolism and correlates with the wide range of cellular functions of these compounds. In this aspect, sphingolipids are involved in almost all cellular functions and networks including cell growth, cell cycle, cell death, inflammation, cell migration and differentiation, autophagy, metabolism, nutrient uptake, angiogenesis, and immune system. One important role of these bioactive lipids is their participation on the regulation of receptor function through the modulation of membrane structure. Although the relation between several metabolic diseases and the loss of function of enzymes directly involve in sphingolipid metabolism has been widely described, growing evidence has shown the impact of sphingolipids on polygenic diseases such as cancer, diabetes, cardiovascular diseases, and neurodegenerative disorders such as Parkinson and Alzheimer. In this sense, due to the global impact of sphingolipids on cell functions, it is important to continue and extend their study by the combination of several omics' sciences, including genomics, proteomics, transcriptomics, epigenomics, metabolomics, and lipidomics, with a holistic view of cell biology through systems biology approaches. This will complete our understanding of sphingolipids metabolism and function that will impact the diagnosis and treatment of several diseases for which sphingolipids represent a key part of their pathophysiology.

Author Contributions: A.F.L., O.Y.E.P., and D.A.S wrote the original draft. A.F.L., S.L.A., C.J.A.D., A.J.E.M, reviewed and edited the manuscript. All authors contributed to the literature analysis. All authors have read and approved the final manuscript.

Funding: C.J.A-D and A.J.E.M are supported by the Ministry of Science, Technology, and Innovation, Colombia (Grant ID 120380763212 - PPTA \# 8352), and by the Pontificia Universidad Javeriana (PPTA \# 8275, 20289, and 20300). A.F.L. received a doctoral scholarship from Pontificia Universidad Javeriana. D.A.S received a young researcher fellowship (Contract 829-2018 - PPTA \#8728) from the Ministry of Science, Technology, and Innovation, Colombia.

Conflicts of Interest: The authors declare no conflict of interest. The funders had no role in the design of the study; in the collection, analyses, or interpretation of data; in the writing of the manuscript, or in the decision to publish the review.

\section{Abbreviations}

\begin{tabular}{|l|l|}
\hline AMPAR & $\alpha$-amino-3-hydroxy-5-methyl-4-isoxazole propionic acid receptor \\
\hline Cer & Ceramide \\
\hline CERT & Ceramide-transfer protein \\
\hline CNS & Central Nervous System \\
\hline ER & Endoplasmic reticulum \\
\hline FAPP2 & Four-phosphate adaptor protein 2 \\
\hline GalCer & Galactosylceramide \\
\hline GlcCer & Glucosylceramide \\
\hline GSL & Glycosphingolipids \\
\hline IP3 & Inositol triphosphate \\
\hline LacCer & Lactosylceramide \\
\hline LTP & Long-Term Potentiation \\
\hline MAG & Myelin-associated glycoprotein \\
\hline MBP & Myelin basic protein \\
\hline NMDARs & N-methyl-D-aspartate receptors \\
\hline ROS & Reactive oxygen species \\
\hline S1P & Sphingosine 1P \\
\hline SM & Sphingomyelin \\
\hline SPC & Sphingosylphosphorylcholine \\
\hline
\end{tabular}




\begin{tabular}{|l|l|}
\hline TNF $\alpha$ & Tumor necrosis factor- $\alpha$ \\
\hline
\end{tabular}




\section{References}

1. Gault, C.R., L.M. Obeid, and Y.A. Hannun, An overview of sphingolipid metabolism: from synthesis to breakdown. Adv Exp Med Biol, 2010. 688: p. 1-23.

2. $\quad$ Ogretmen, B., Sphingolipid metabolism in cancer signalling and therapy. Nat Rev Cancer, 2018. 18(1): p. 3350.

3. Bouscary, A., et al., Sphingolipids metabolism alteration in the central nervous system: Amyotrophic lateral sclerosis (ALS) and other neurodegenerative diseases. Semin Cell Dev Biol, 2020.

4. Dasgupta, S. and S.K. Ray, Diverse Biological Functions of Sphingolipids in the CNS: Ceramide and Sphingosine Regulate Myelination in Developing Brain but Stimulate Demyelination during Pathogenesis of Multiple Sclerosis. J Neurol Psychol, 2017. 5(1).

5. Parashuraman, S. and G. D'Angelo, Visualizing sphingolipid biosynthesis in cells. Chem Phys Lipids, 2019. 218: p. 103-111.

6. Sandhoff, R. and K. Sandhoff, Emerging concepts of ganglioside metabolism. FEBS Lett, 2018. 592(23): p. 3835-3864.

7. Leal, A.F., et al., GM2 Gangliosidoses: Clinical Features, Pathophysiological Aspects, and Current Therapies. Int J Mol Sci, 2020. 21(17).

8. Olsen, A.S.B. and N.J. Færgeman, Sphingolipids: membrane microdomains in brain development, function and neurological diseases. Open Biol, 2017. 7(5).

9. Kwak, D.H., et al., Roles of gangliosides in mouse embryogenesis and embryonic stem cell differentiation. Exp Mol Med, 2011. 43(7): p. 379-88.

10. Dasgupta, S., et al., Neutral monoglycosylceramides in rat brain: occurrence, molecular expression and developmental variation. Dev Neurosci, 1997. 19(2): p. 152-61.

11. O'Brien, J.S. and E.L. Sampson, Lipid composition of the normal human brain: gray matter, white matter, and myelin. J Lipid Res, 1965. 6(4): p. 537-44.

12. Schnaar, R.L., The Biology of Gangliosides. Adv Carbohydr Chem Biochem, 2019. 76: p. 113-148.

13. Vyas, A.A., et al., Gangliosides are functional nerve cell ligands for myelin-associated glycoprotein (MAG), an inhibitor of nerve regeneration. Proc Natl Acad Sci U S A, 2002. 99(12): p. 8412-7.

14. Kuhn, S., et al., Oligodendrocytes in Development, Myelin Generation and Beyond. Cells, 2019. 8(11).

15. Davis, D.L., et al., Dynamics of sphingolipids and the serine palmitoyltransferase complex in rat oligodendrocytes during myelination. J Lipid Res, 2020. 61(4): p. 505-522.

16. Xu, Y., Y. Ramu, and Z. Lu, Removal of phospho-head groups of membrane lipids immobilizes voltage sensors of K+ channels. Nature, 2008. 451(7180): p. 826-9.

17. Ledeen, R.W. and G. Wu, Ganglioside function in calcium homeostasis and signaling. Neurochem Res, 2002. 27(7-8): p. 637-47.

18. Chen, N., et al., Ganglioside/calmodulin kinase II signal inducing cdc42-mediated neuronal actin reorganization. Neuroscience, 2003. 120(1): p. 163-76.

19. Furuya, S., K. Ono, and Y. Hirabayashi, Sphingolipid biosynthesis is necessary for dendrite growth and survival of cerebellar Purkinje cells in culture. J Neurochem, 1995. 65(4): p. 1551-61.

20. Furuya, S., et al., Ceramide and its interconvertible metabolite sphingosine function as indispensable lipid factors involved in survival and dendritic differentiation of cerebellar Purkinje cells. J Neurochem, 1998. 71(1): p. 36677.

21. Tabatadze, N., et al., Inhibition of neutral sphingomyelinase-2 perturbs brain sphingolipid balance and spatial memory in mice. J Neurosci Res, 2010. 88(13): p. 2940-51. 
22. Platt, F.M., Sphingolipid lysosomal storage disorders. Nature, 2014. 510(7503): p. 68-75.

23. Pakanova, Z., et al., Inherited metabolic disorders of glycoconjugate metabolism. Bratisl Lek Listy, 2020. 121(10): p. 760-766.

24. Valle DL, A.S., et al. The Online Metabolic and Molecular Bases of Inherited Disease. 2019 [cited 2020 November 20th].

25. Aerts, J.M.F.G., et al., Glycosphingolipids and lysosomal storage disorders as illustrated by gaucher disease. Curr Opin Chem Biol, 2019. 53: p. 204-215.

26. Hannun, Y.A. and L.M. Obeid, Sphingolipids and their metabolism in physiology and disease. Nat Rev Mol Cell Biol, 2018. 19(3): p. 175-191.

27. Giordano, G., Ceramide-transfer protein-mediated ceramide transfer is a structurally tunable flow-inducing mechanism with structural feed-forward loops. R Soc Open Sci, 2018. 5(6): p. 180494.

28. Kumagai, K. and K. Hanada, Structure, functions and regulation of CERT, a lipid-transfer protein for the delivery of ceramide at the ER-Golgi membrane contact sites. FEBS Lett, 2019. 593(17): p. 2366-2377.

29. Yamaji, T. and K. Hanada, Sphingolipid metabolism and interorganellar transport: localization of sphingolipid enzymes and lipid transfer proteins. Traffic, 2015. 16(2): p. 101-22.

30. D'Angelo, G., et al., Vesicular and non-vesicular transport feed distinct glycosylation pathways in the Golgi. Nature, 2013. 501(7465): p. 116-20.

31. Chiricozzi, E., et al., GM1 Ganglioside Is A Key Factor in Maintaining the Mammalian Neuronal Functions Avoiding Neurodegeneration. Int J Mol Sci, 2020. 21(3).

32. Jiang, L., et al., Effects of gangliosides on the activity of the plasma membrane Ca2+-ATPase. Biochim Biophys Acta, 2014. 1838(5): p. 1255-65.

33. Stern, C.A. and M. Tiemeyer, A Ganglioside-Specific Sialyltransferase Localizes to Axons and Non-Golgi Structures in Neurons. Journal of Neuroscience, 2001. 21: p. 1434-1443.

34. Preti, A., et al., Occurrence of sialyltransferase activity in the synaptosomal membranes prepared from calf brain cortex. J Neurochem, 1980. 35(2): p. 281-96.

35. Saito, M., et al., Characterization of sialidase activity in mouse synaptic plasma membranes and its age-related changes. J Neurosci Res, 1995. 40(3): p. 401-6.

36. Miyagi, T., et al., Molecular cloning and characterization of a plasma membrane-associated sialidase specific for gangliosides. J Biol Chem, 1999. 274(8): p. 5004-11.

37. Mencarelli, S., et al., Identification of plasma membrane associated mature beta-hexosaminidase A, active towards GM2 ganglioside, in human fibroblasts. FEBS Lett, 2005. 579(25): p. 5501-6.

38. Aureli, M., et al., Activity of plasma membrane beta-galactosidase and beta-glucosidase. FEBS Lett, 2009. 583(15): p. 2469-73.

39. Rodriguez, J.A., et al., Plasma membrane ganglioside sialidase regulates axonal growth and regeneration in hippocampal neurons in culture. J Neurosci, 2001. 21(21): p. 8387-95.

40. Miranda, A.M., et al., Differential lipid composition and regulation along the hippocampal longitudinal axis. Transl Psychiatry, 2019. 9(1): p. 144.

41. $\mathrm{Yu}, \mathrm{Q}$. , et al., Lipidome alterations in human prefrontal cortex during development, aging, and cognitive disorders. Mol Psychiatry, 2020. 25(11): p. 2952-2969.

42. Svennerholm, L. and M.T. Vanier, The distribution of lipids in the human nervous system. 3. Fatty acid composition of phosphoglycerides of human foetal and infant brain. Brain Res, 1973. 50(2): p. 341-51.

43. Kracun, I., et al., Human brain gangliosides in development, aging and disease. Int J Dev Biol, 1991. 35(3): p. 289-95. 
44. Schnaar, R.L. and P.H. Lopez, Myelin-associated glycoprotein and its axonal receptors. J Neurosci Res, 2009. 87(15): p. 3267-76.

45. Saadat, L., et al., Absence of oligodendroglial glucosylceramide synthesis does not result in CNS myelin abnormalities or alter the dysmyelinating phenotype of CGT-deficient mice. Glia, 2010. 58(4): p. 391-8.

46. Camargo, N., et al., Oligodendroglial myelination requires astrocyte-derived lipids. PLoS Biol, 2017. 15(5): p. e1002605.

47. van Echten-Deckert, G. and T. Herget, Sphingolipid metabolism in neural cells. Biochim Biophys Acta, 2006. 1758(12): p. 1978-94.

48. Riboni, L., et al., Cultured granule cells and astrocytes from cerebellum differ in metabolizing sphingosine. J Neurochem, 2000. 75(2): p. 503-10.

49. Riboni, L., G. Tettamanti, and P. Viani, Ceramide in primary astrocytes from cerebellum: metabolism and role in cell proliferation. Cerebellum, 2002. 1(2): p. 129-35.

50. Bieberich, E., Ceramide and sphingosine-1-phosphate signaling in embryonic stem cell differentiation. Methods Mol Biol, 2012. 874: p. 177-92.

51. Wang, P., et al., Roles of sphingosine-1-phosphate signaling in cancer. Cancer Cell Int, 2019. 19: p. 295.

52. de Wit, N.M., et al., Astrocytic ceramide as possible indicator of neuroinflammation. J Neuroinflammation, 2019. 16(1): p. 48.

53. Assi, E., et al., Sphingolipids and brain resident macrophages in neuroinflammation: an emerging aspect of nervous system pathology. Clin Dev Immunol, 2013. 2013: p. 309302.

54. Mencarelli, C. and P. Martinez-Martinez, Ceramide function in the brain: when a slight tilt is enough. Cell Mol Life Sci, 2013. 70(2): p. 181-203.

55. Lee, J.Y., H.K. Jin, and J.S. Bae, Sphingolipids in neuroinflammation: a potential target for diagnosis and therapy. BMB Rep, 2020. 53(1): p. 28-34.

56. Wang, J., et al., Ganglioside GD3 is required for neurogenesis and long-term maintenance of neural stem cells in the postnatal mouse brain. J Neurosci, 2014. 34(41): p. 13790-800.

57. Wang, J. and R.K. Yu, Interaction of ganglioside GD3 with an EGF receptor sustains the self-renewal ability of mouse neural stem cells in vitro. Proc Natl Acad Sci U S A, 2013. 110(47): p. 19137-42.

58. Panzetta, P., H.J. Maccioni, and R. Caputto, Synthesis of retinal gangliosides during chick embryonic development. J Neurochem, 1980. 35(1): p. 100-8.

59. Purpura, D.P. and K. Suzuki, Distortion of neuronal geometry and formation of aberrant synapses in neuronal storage disease. Brain Res, 1976. 116(1): p. 1-21.

60. Walkley, S.U., M. Zervas, and S. Wiseman, Gangliosides as modulators of dendritogenesis in normal and storage disease-affected pyramidal neurons. Cereb Cortex, 2000. 10(10): p. 1028-37.

61. Harel, R. and A.H. Futerman, Inhibition of sphingolipid synthesis affects axonal outgrowth in cultured hippocampal neurons. J Biol Chem, 1993. 268(19): p. 14476-81.

62. Da Silva, J.S., et al., Asymmetric membrane ganglioside sialidase activity specifies axonal fate. Nat Neurosci, 2005. 8(5): p. 606-15.

63. Mendez-Otero, R. and M.F. Santiago, Functional role of a specific ganglioside in neuronal migration and neurite outgrowth. Braz J Med Biol Res, 2003. 36(8): p. 1003-13.

64. Yoon, H., et al., Interplay between exercise and dietary fat modulates myelinogenesis in the central nervous system. Biochim Biophys Acta, 2016. 1862(4): p. 545-555.

65. Pan, B., et al., Myelin-associated glycoprotein and complementary axonal ligands, gangliosides, mediate axon stability in the CNS and PNS: neuropathology and behavioral deficits in single-and double-null mice. Exp Neurol, 2005. 195(1): p. 208-17. 
66. DeBruin, L.S., et al., Developmental partitioning of myelin basic protein into membrane microdomains. J Neurosci Res, 2005. 80(2): p. 211-25.

67. Boggs, J.M., et al., Participation of galactosylceramide and sulfatide in glycosynapses between oligodendrocyte or myelin membranes. FEBS Lett, 2010. 584(9): p. 1771-8.

68. Ben-David, O., et al., Encephalopathy caused by ablation of very long acyl chain ceramide synthesis may be largely due to reduced galactosylceramide levels. J Biol Chem, 2011. 286(34): p. 30022-33.

69. Susuki, K., et al., Gangliosides contribute to stability of paranodal junctions and ion channel clusters in myelinated nerve fibers. Glia, 2007. 55(7): p. 746-57.

70. Chiavegatto, S., et al., A functional role for complex gangliosides: motor deficits in GM2/GD2 synthase knockout mice. Exp Neurol, 2000. 166(2): p. 227-34.

71. Sonnino, S. and A. Prinetti, The role of sphingolipids in neuronal plasticity of the brain. J Neurochem, 2016. 137(4): p. 485-8.

72. Wheeler, D., et al., Tumor necrosis factor-alpha-induced neutral sphingomyelinase-2 modulates synaptic plasticity by controlling the membrane insertion of NMDA receptors. J Neurochem, 2009. 109(5): p. 1237-49.

73. Fujii, S., et al., Effects of the mono- and tetrasialogangliosides GM1 and GQ1b on ATP-induced long-term potentiation in hippocampal CA1 neurons. Glycobiology, 2002. 12(5): p. 339-44.

74. Hanley, J.G., Subunit-specific trafficking mechanisms regulating the synaptic expression of Ca(2+)-permeable AMP A receptors. Semin Cell Dev Biol, 2014. 27: p. 14-22.

75. Prendergast, J., et al., Ganglioside regulation of AMPA receptor trafficking. J Neurosci, 2014. 34(39): p. 1324658.

76. Milescu, M., et al., Interactions between lipids and voltage sensor paddles detected with tarantula toxins. Nat Struct Mol Biol, 2009. 16(10): p. 1080-5.

77. Martens, J.R., et al., Isoform-specific localization of voltage-gated K+ channels to distinct lipid raft populations. Targeting of Kv1.5 to caveolae. J Biol Chem, 2001. 276(11): p. 8409-14.

78. Wong, W. and L.C. Schlichter, Differential recruitment of Kv1.4 and Kv4.2 to lipid rafts by PSD-95. J Biol Chem, 2004. 279(1): p. 444-52.

79. Colombaioni, L. and M. Garcia-Gil, Sphingolipid metabolites in neural signalling and function. Brain Res Brain Res Rev, 2004. 46(3): p. 328-55.

80. Buzas, B., Regulation of nociceptin/orphanin FQ gene expression in astrocytes by ceramide. Neuroreport, 2002. 13(14): p. 1707-10.

81. Ghosh, T.K., J. Bian, and D.L. Gill, Intracellular calcium release mediated by sphingosine derivatives generated in cells. Science, 1990. 248(4963): p. 1653-6.

82. Lloyd-Evans, E., et al., Glucosylceramide and glucosylsphingosine modulate calcium mobilization from brain microsomes via different mechanisms. J Biol Chem, 2003. 278(26): p. 23594-9.

83. Lloyd-Evans, E., et al., Lyso-glycosphingolipids mobilize calcium from brain microsomes via multiple mechanisms. Biochem J, 2003. 375(Pt 3): p. 561-5.

84. Blázquez, C., I. Galve-Roperh, and M. Guzmán, De novo-synthesized ceramide signals apoptosis in astrocytes via extracellular signal-regulated kinase. FASEB J, 2000. 14(14): p. 2315-22.

85. Stoica, B.A., et al., Ceramide-induced neuronal apoptosis is associated with dephosphorylation of Akt, BAD, FKHR, GSK-3beta, and induction of the mitochondrial-dependent intrinsic caspase pathway. Mol Cell Neurosci, 2003. 22(3): p. 365-82.

86. France-Lanord, V., et al., Mitochondrial free radical signal in ceramide-dependent apoptosis: a putative mechanism for neuronal death in Parkinson's disease. J Neurochem, 1997. 69(4): p. 1612-21. 
87. García-Ruiz, C., et al., Direct effect of ceramide on the mitochondrial electron transport chain leads to generation of reactive oxygen species. Role of mitochondrial glutathione. J Biol Chem, 1997. 272(17): p. 11369-77.

88. Raisova, M., et al., Bcl-2 overexpression prevents apoptosis induced by ceramidase inhibitors in malignant melanoma and HaCaT keratinocytes. FEBS Lett, 2002. 516(1-3): p. 47-52.

89. Virgolini, M.J., et al., Neurite atrophy and apoptosis mediated by PERK signaling after accumulation of GM2ganglioside. Biochim Biophys Acta Mol Cell Res, 2019. 1866(2): p. 225-239.

90. Bachis, A., et al., Gangliosides prevent excitotoxicity through activation of TrkB receptor. Neurotox Res, 2002. 4(3): p. 225-34.

91. Harada, J., et al., Sphingosine-1-phosphate induces proliferation and morphological changes of neural progenitor cells. J Neurochem, 2004. 88(4): p. 1026-39.

92. Meng, H., Y. Yuan, and V.M. Lee, Loss of sphingosine kinase 1/S1P signaling impairs cell growth and survival of neurons and progenitor cells in the developing sensory ganglia. PLoS One, 2011. 6(11): p. e27150.

93. Ryckman, A.E., I. Brockhausen, and J.S. Walia, Metabolism of Glycosphingolipids and Their Role in the Pathophysiology of Lysosomal Storage Disorders. Int J Mol Sci, 2020. 21(18).

94. Polo, G., et al., Plasma and dried blood spot lysosphingolipids for the diagnosis of different sphingolipidoses: a comparative study. Clin Chem Lab Med, 2019. 57(12): p. 1863-1874.

95. Anderson, S., Newborn Screening for Lysosomal Storage Disorders. J Pediatr Health Care, 2018. 32(3): p. 285-294.

96. Li, D., et al., Early prenatal diagnosis of lysosomal storage disorders by enzymatic and molecular analysis. Prenat Diagn, 2018. 38(10): p. 779-787.

97. Zanetti, A., et al., Molecular diagnosis of patients affected by mucopolysaccharidosis: a multicenter study. Eur J Pediatr, 2019. 178(5): p. 739-753.

98. Pelled, D., et al., Inhibition of calcium uptake via the sarco/endoplasmic reticulum Ca2+-ATPase in a mouse model of Sandhoff disease and prevention by treatment with N-butyldeoxynojirimycin. J Biol Chem, 2003. 278(32): p. 29496-501.

99. Ginzburg, L., et al., An exposed carboxyl group on sialic acid is essential for gangliosides to inhibit calcium uptake via the sarcolendoplasmic reticulum Ca2+-ATPase: relevance to gangliosidoses. J Neurochem, 2008. 104(1): p. 140-6.

100. Wada, R., C.J. Tifft, and R.L. Proia, Microglial activation precedes acute neurodegeneration in Sandhoff disease and is suppressed by bone marrow transplantation. Proc Natl Acad Sci U S A, 2000. 97(20): p. 10954-9.

101. Bradbury, A.M., et al., AAV-mediated gene delivery attenuates neuroinflammation in feline Sandhoff disease. Neuroscience, 2017. 340: p. 117-125.

102. Cachon-Gonzalez, M.B., E. Zaccariotto, and T.M. Cox, Genetics and Therapies for GM2 Gangliosidosis. Curr Gene Ther, 2018. 18(2): p. 68-89.

103. Jeyakumar, M., et al., Central nervous system inflammation is a hallmark of pathogenesis in mouse models of GM1 and GM2 gangliosidosis. Brain, 2003. 126(Pt 4): p. 974-87.

104. Ogawa, Y., et al., Inhibition of astrocytic adenosine receptor A. Neurobiol Dis, 2018. 118: p. 142-154.

105. Ogawa, Y., et al., FcR $\gamma$-dependent immune activation initiates astrogliosis during the asymptomatic phase of Sandhoff disease model mice. Sci Rep, 2017. 7: p. 40518.

106. Domingues, H.S., et al., Oligodendrocyte, Astrocyte, and Microglia Crosstalk in Myelin Development, Damage, and Repair. Front Cell Dev Biol, 2016. 4: p. 71.

107. Liddelow, S.A. and B.A. Barres, Reactive Astrocytes: Production, Function, and Therapeutic Potential. Immunity, 2017. 46(6): p. 957-967. 
108. Brekk, O.R., et al., Upregulating $\beta$-hexosaminidase activity in rodents prevents $\alpha$-synuclein lipid associations and protects dopaminergic neurons from $\alpha$-synuclein-mediated neurotoxicity. Acta Neuropathol Commun, 2020. 8(1): p. 127.

109. Cachón-González, M.B., et al., Reversibility of neuropathology in Tay-Sachs-related diseases. Hum Mol Genet, 2014. 23(3): p. 730-48.

110. Suzuki, K., et al., Accumulated $\alpha$-synuclein affects the progression of GM2 gangliosidoses. Exp Neurol, 2016. 284(Pt A): p. 38-49.

111. Bode, H., et al., HSAN1 mutations in serine palmitoyltransferase reveal a close structure-function-phenotype relationship. Hum Mol Genet, 2016. 25(5): p. 853-65.

112. Hornemann, T., et al., The SPTLC3 subunit of serine palmitoyltransferase generates short chain sphingoid bases. J Biol Chem, 2009. 284(39): p. 26322-30.

113. Harmon, J.M., et al., Topological and functional characterization of the SSSPTs, small activating subunits of serine palmitoyltransferase. J Biol Chem, 2013. 288(14): p. 10144-10153. 